\title{
Alveolar bone remodeling and development after immediate orthodontic root movement
}

\begin{abstract}
Introduction: Adult orthodontics is rapidly expanding primarily due to increased patient esthetic and functional demands. Diagnosis and treatment planning for a complex adult case frequently requires the successful integration of a variety of dental disciplines to insure the best possible outcome. This case report illustrates the highly successfully use of orthodontics as the primary discipline towards the correction of esthetic concerns and dentoalveolar deficiencies.

Case report: The patient who is a 37-year-old female with esthetic and functional concerns successfully completes orthodontic treatment as the primary portion of a complex adult restorative case. Post treatment clinical and radiographic results show dramatic esthetic and functional improvement with the elimination of a maxillary anterior crossbite, improvement of a dimensionally constricted maxilla, and dentoalveolar improvements for surgical placement and subsequent restoration of dental implants.

Conclusion: This case report demonstrates the potential of successfully utilizing primary orthodontic treatment in a complex adult case by utilizing a new technology system of braces that facilitates the continuation of eruption while inducing alveolar bone remodeling and development. In addition, this case report provides radiographic and clinical validation of favorable alveolar remodeling and development.
\end{abstract}

Keywords: alveolar bone remodeling and development, interdisciplinary dentistry, orthodontics, fastbraces ${ }^{\circledR}$
Volume 6 Issue 2 - 2017

\author{
Anthony DViazis,' 'Mongkol \\ Thaveeprungsiporn, ${ }^{2}$ Tom C Pagonis ${ }^{3}$ \\ 'Orthodontist, Private Practice, USA \\ ${ }^{2}$ Private Practice, Greece \\ ${ }^{3}$ Department of Restorative Dentistry and Biomaterials \\ Sciences, Harvard School of Dental Medicine, USA
}

Correspondence: Tom C Pagonis, Department of Restorative Dentistry and Biomaterials Sciences, Harvard School of Dental Medicine, 188 Longwood Avenue, Boston, MA 021 I5, USA, Email dental.research@comcast.net

Received: January 26, 2017 | Published: January 31, 2017

\section{Introduction}

Orthodontic treatment of the adult patient has grown substantially in recent years primarily due to the longevity of natural dentition, an increased awareness of functional benefits of orthodontics and increasing esthetic demands. ${ }^{1}$ Compared to the younger patient and in addition to possible malalignment of teeth and jaws, the adult patient will more likely present with a compromised dentition that could include missing teeth, increased wear of teeth along with accompanying periodontal and endodontic involvement. For a complex adult case, careful coordination among disciplines is critically important in order to optimize treatment results. ${ }^{2,3}$ In these complex adult clinical situations, orthodontic treatment requires different bio-mechanical strategies for tooth movement. The crowntipping mechanics of round wires of older orthodontic systems present treatment challenges for adult non-extraction therapy because these systems can't rely on growth potential as compared to a younger patient and they are unable to leverage the osteogenic potential of alveolar bone. In 2014, Viazis et al. ${ }^{4}$ proposed new, disease-based orthodontic terminology for malpositioned teeth based on the clinical morphology, appearance and contour of the alveolar bone and ridge. This diagnosis, which is called orthodontosis, is defined as the deficiency of the alveolar bone in the horizontal dimension caused by the displaced root(s) of the tooth, typically palatally or lingually. This case report illustrates the successful and efficient utilization of a new technology orthodontic system of braces which facilitates alveolar bone remodeling and development by uprighting the roots of malpostioned teeth immediately at the onset of treatment and with light forces which may simulate natural eruption forces. This action induces alveolar bone remodeling and development horizontally and at approximately the apical one half of the root. The clinical manifestation is the elimination of this non-inflammatory deficiency or negative architecture resulting in the correction of malalignment of teeth for esthetic, surgical and restorative purposes.

\section{Case report}

\section{Diagnosis}

The patient is a 37-year-old female who presented to the second author's private practice in Bangkok, Thailand with a chief complaint of esthetic concerns with her smile and with difficulty chewing (Figure 1). On examination the patient has a mesoproscopic facial form, with slight rotation/crowding of mandibular right incisors and a relatively narrow maxillary arch with her maxillary right canine and lateral incisor in crossbite. Functionally, the patient only occludes up to her first molar on the right and is missing both her mandibular right second molar along with her mandibular left first molar. The maxillary right second and third molars have hyper erupted into the opposing edentulous space and the mandibular left second molar has drifted into the adjacent mandibular first molar space.

\section{Treatment objectives}

A review of pre-treatment panoramic and lateral cephalogram radiographs confirm clinical findings (Figure 2) and pre-treatment cone beam computed tomography (CBCT) cross sectional images through the maxillary right central incisor, was measured buccallingually to quantify pre-treatment alveolar bone dimensions (Figure 3). In addition, CBCT imaging was completed for the edentulous mandibular left first molar and mandibular right second molar for 
placement of dental implants. The patient was treatment planned for extraction of the hyper erupted and unopposed maxillary right third molar along with comprehensive orthodontic treatment using the orthodontic system Fastbraces ${ }^{\circledR}$ in order to eliminate a maxillary incisor crossbite and to upright the mandibular left second molar before dental implant placement in the adjacent first molar space. Both implants were to be restored after the completion of orthodontic treatment. The patient was initially apprehensive of the longevity of orthodontic treatment but agreed to treatment with Fastbraces ${ }^{\circledR}$ because of the reduced estimated treatment time. Before the start of comprehensive orthodontic treatment, a Hiossen (Fairless Hills, PA, USA) (D5.0/L 10mm) dental implant was placed in the edentulous right second mandibular molar area following incision and flap reflection. A healing abutment was placed and the site was sutured. In the same appointment, the maxillary right third molar was extracted.

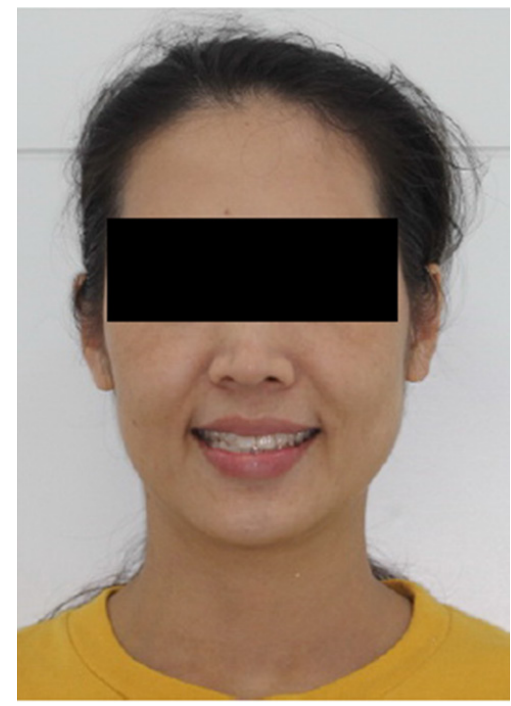

Figure IA Pre-treatment facial and intraoral frontal view photographs.
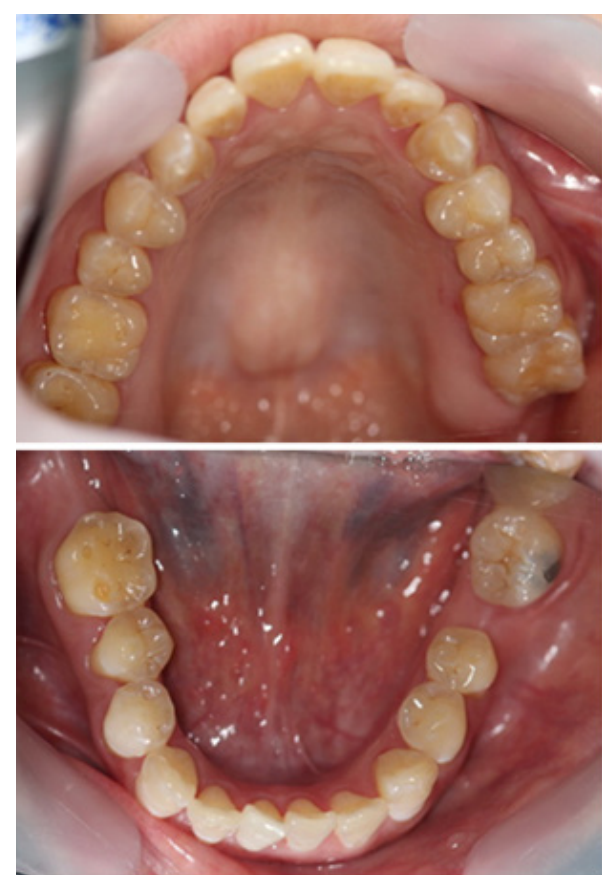

Figure IB Pre-treatment intraoral occlusal view photographs.
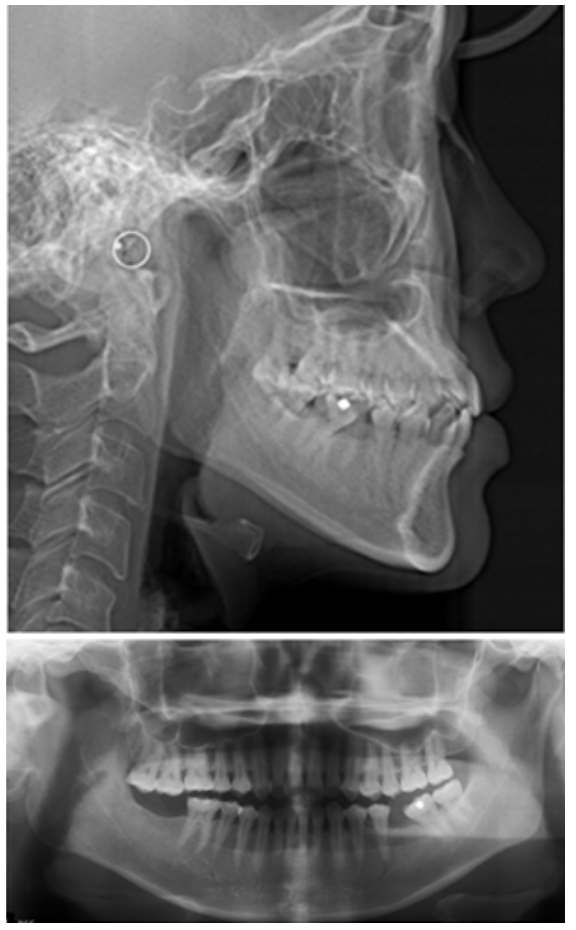

Figure 2 Pre-treatment lateral cephalogram and panoramic radiographs.

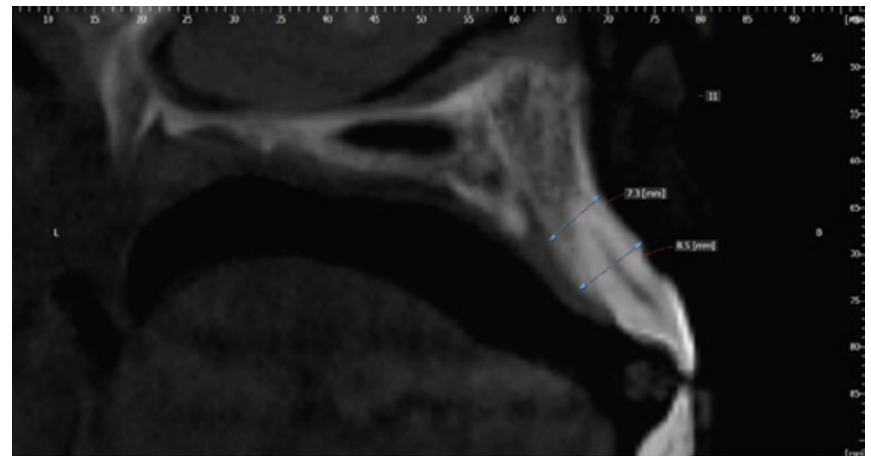

Figure 3 Pre-treatment cross-sectional $\mathrm{CBCT}$ of maxillary right central incisor.

\section{Treatment progress}

About 20 days later and after post-operative healing of the extraction site and implant placement in the mandibular right second molar area, orthodontic treatment was initiated with strategic interproximal reduction (IPR) of rotated/crowded mandibular right incisor teeth in proximal contact. In addition, glass ionomer was placed on the occlusal surfaces of the mandibular right first molar and mandibular left second molar to open the bite for optimal posttreatment occlusion. Brackets were placed on all teeth while skipping the maxillary left first molar because of distolingual rotation. A fixed button was placed on the lingual surface of the maxillary right canine with elastics placed for patient use for almost 24 hours per day (Figure 4). Specifically, elastic from the lingual button of the maxillary right canine to labial side of the mandibular right canine and first premolar was prescribed in order to apply lingual force to the maxillary right canine in order to tip the canine labially and out of crossbite. Elastics were also placed on the left from the maxillary left first premolar to the mandibular left canine and first premolar (Figure 5). The patient 
was seen about 20 days later at which time the maxillary first molar was orthodontically engaged and ligature wires were secured from the mandibular left canine to the mandibular left second premolar and mandibular left canine to the mandibular left first molar. The patient was instructed to continue wearing the elastics in the same fashion. About 20 days later the patient returned for her third appointment and ligature ties were placed from the maxillary right canine to the maxillary left canine along with the placement of a mandibular elastic chain from the mandibular left lateral incisor to the mandibular left second premolar. In addition, the remaining glass ionomer was removed from the mandibular molars. About 14 days later the patient returned and a panoramic radiograph was taken to confirm root parallelism and to evaluate the uprighting of the mandibular left second molar (Figure 6). Additional strategic IPR was performed between mandibular central incisors and repositioning of the bracket mesially and gingivally was completed for the mandibular left second molar to facilitate uprighting about 14 days later during the fifth appointment. At the sixth appointment or at about the 90th day of treatment the mandibular wire was temporarily removed and a Hiossen dental implant (D5.0/L $10 \mathrm{~mm}$ ) was placed in the mandibular left first molar site in a similar manner. The site was grafted for guided bone regeneration with Osteon $^{\mathrm{TM}}$ (Genoss, Gyeonggi-do, Korea) plus autologous fibrin glue (AFG) and concentrated growth factors from staged centrifugation of the patient's blood (Figure 7). A healing abutment was placed and site was sutured. At the seventh appointment and or at about the 120th day of treatment the patient was very satisfied with esthetic results and ligature was secured from maxillary first molar to the opposite maxillary first molar (Figure 8). At the eighth appointment or around the 150th day of treatment, all braces were removed and impressions made for the mandibular implant crowns. The permanent (Zirconia) crowns for these implants were cemented a week later (Figure 9) and impressions for clear maxillary and mandibular retainers were made. The following week the patient returned for insertion and delivery of maxillary and mandibular retainers.

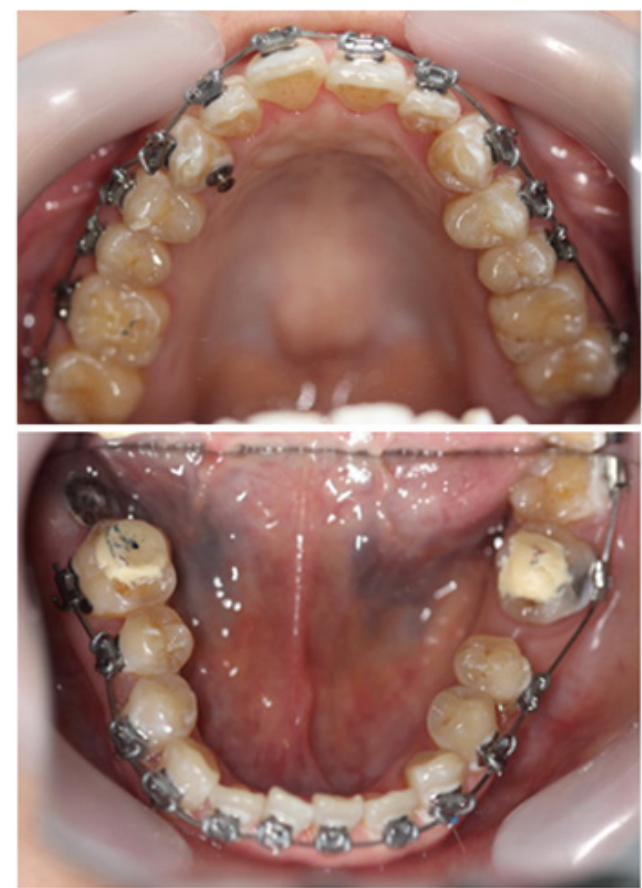

Figure 4 Intra-oral maxillary and mandibular arches immediately after placement of braces.

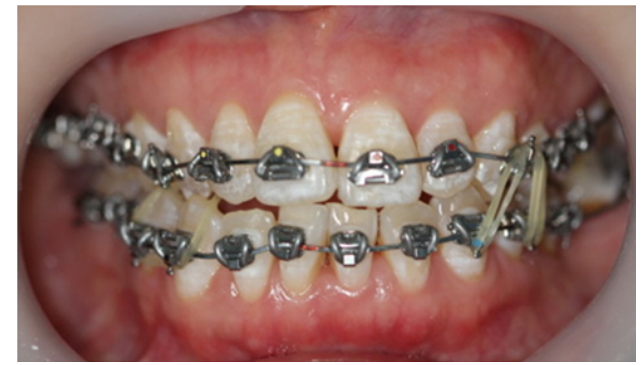

Figure 5 Frontal view immediately after placement of braces on maxillary and mandibular teeth with addition of elastics.

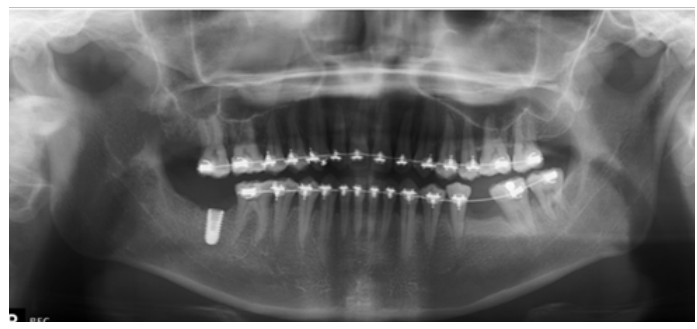

Figure 6 Panoramic radiograph two months after starting Fastbraces $\mathbb{R}$ showing up righting of the mandibular left second molar with healing of maxillary first molar extraction site and dental implant fixture to replace the mandibular right second molar.
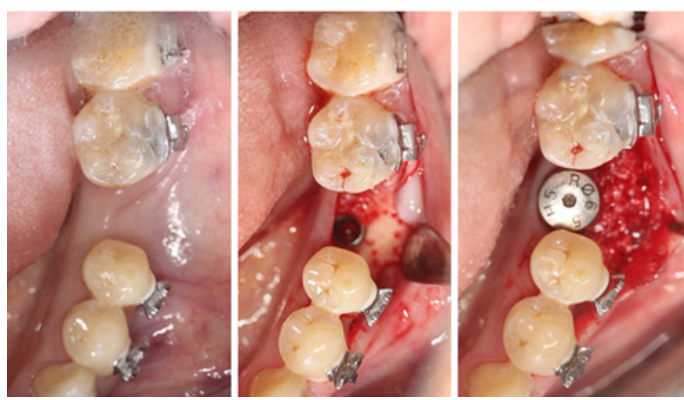

Figure 7 Hiossen implant placement with guided bone regeneration in mandibular left molar site.

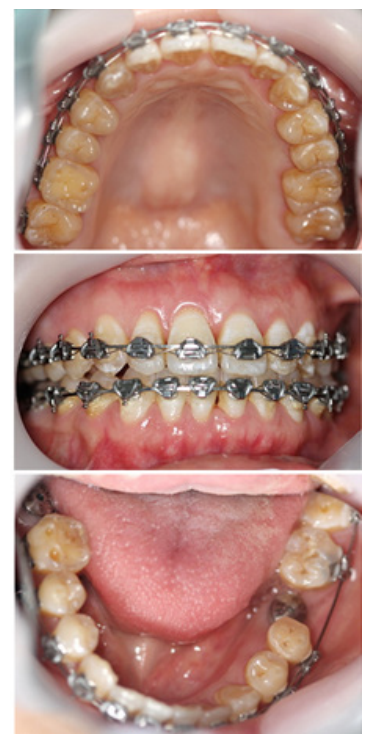

Figure 8 Post-treatment intraoral frontal and occlusal photographs immediately before removal of braces. 

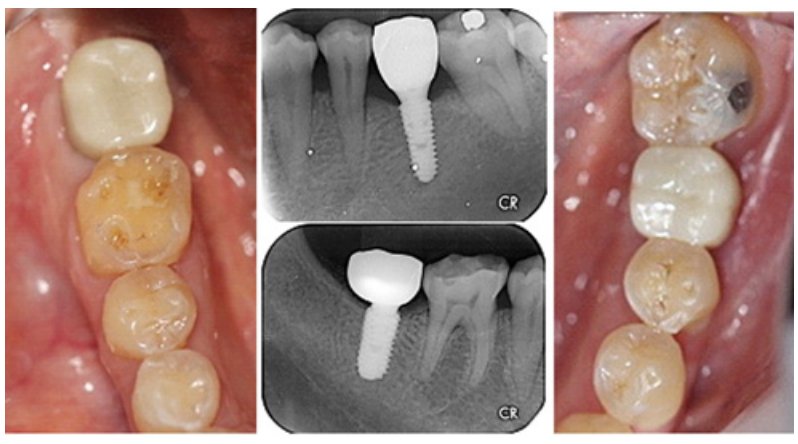

Figure 9 Post-treatment insertion and intraoral periapical radiographs of mandibular implant crowns.

\section{Treatment and results}

Clinical results along with photographs and post-treatment radiographs (Figure 10) including CBCT images comparing pre and post-treatment show dramatic_esthetic and functional improvement, the elimination of maxillary anterior crossbite (Figure 11), expansion of the constricted maxillary arch, uprighting of the left mandibular second molar, successful osseointegration with permanent restoration of mandibular dental implants and correction of anterior guidance with a stable occlusion (Figure 12). Of note is the radiographic evidence of alveolar bone development by comparing the before and after alveolar bone dimension of cross-sectional CBCT images in the right maxillary central incisor. In addition, the images clearly show the reversal of alveolar bone hypoplasia or negative architecture associated with orthodontosis into normal bone and normal architecture (Figure 13).
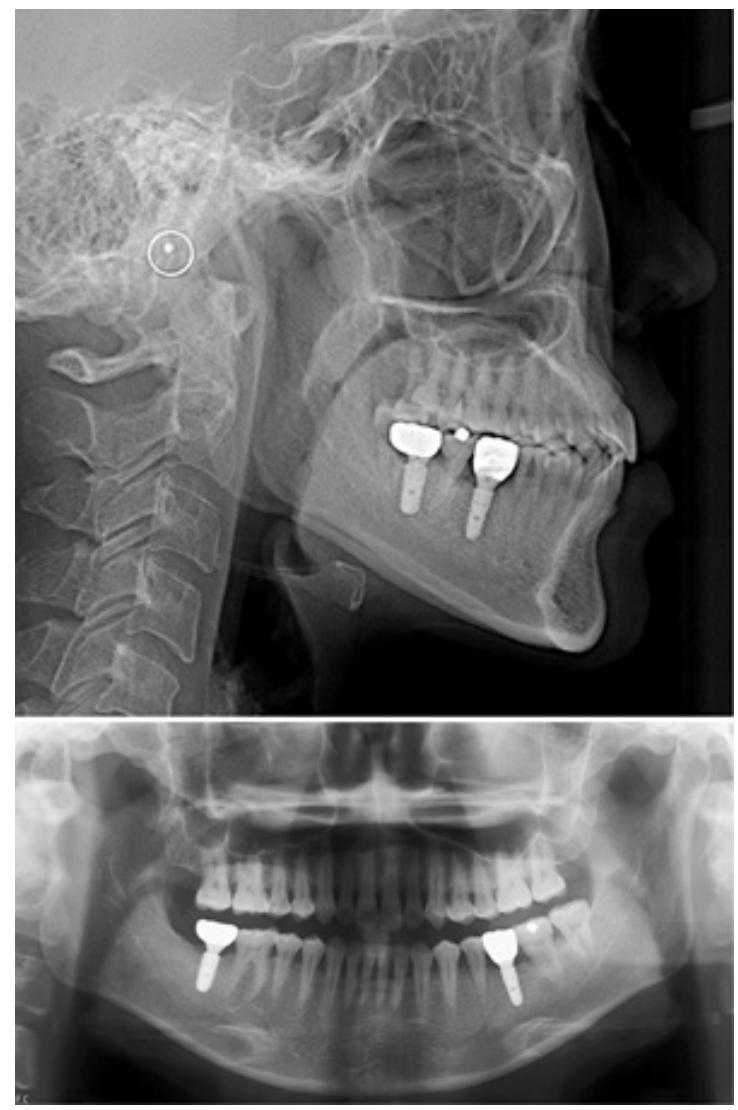

Figure 10 Post-treatment lateral cephalogram and panoramic radiographs.

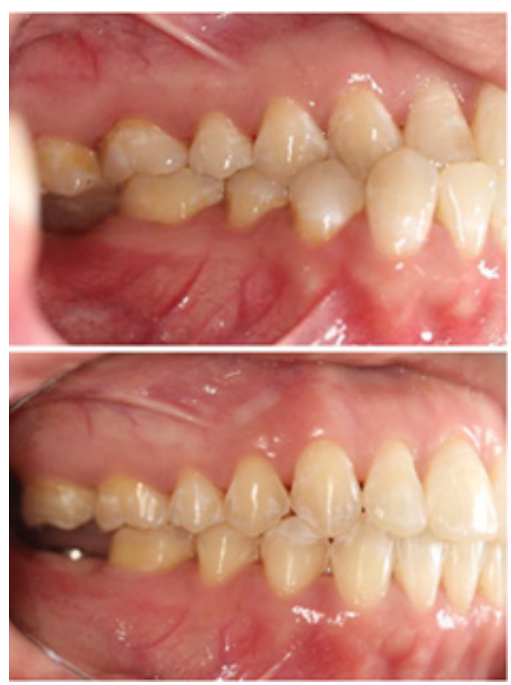

Figure I I Pre and post-treatment intraoral photographs showing elimination of anterior crossbite.
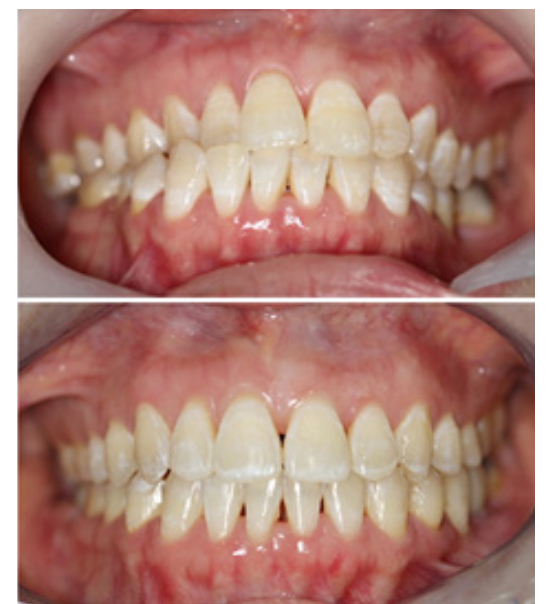

Figure I 2A Pre and post-treatment intraoral frontal photographs.
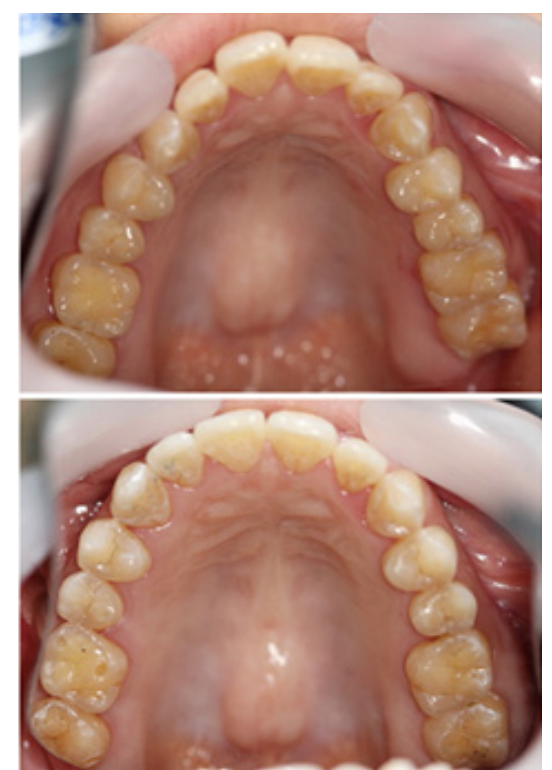

Figure I2B Pre and post-treatment intraoral maxillary arch photographs. 

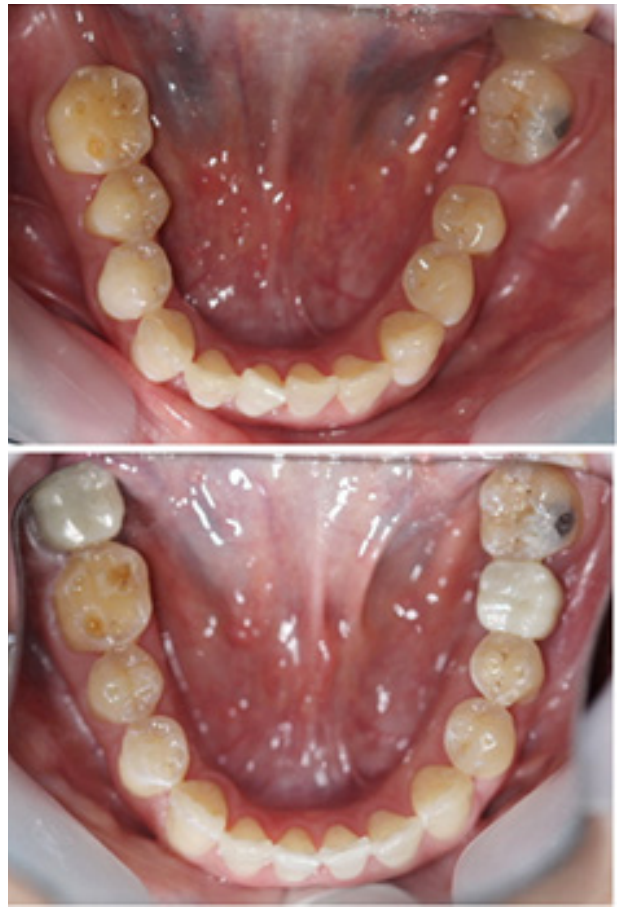

Figure I2C Pre and post-treatment intraoral mandibular arch photographs.
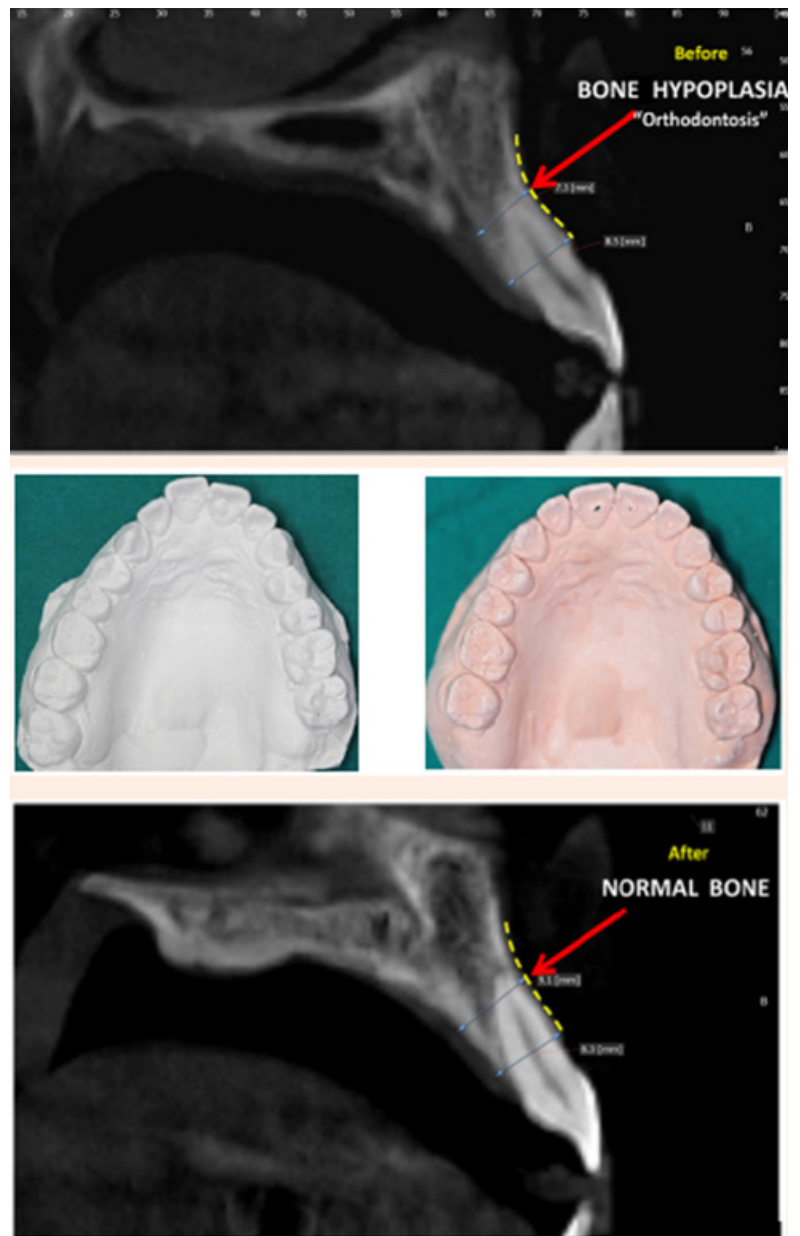

Figure 13 Comparing before and after CBCT cross-sectional image of maxillary right central incisor and maxillary casts.

\section{Discussion}

The complex adult dental case requires the expertise and skills of different specialties sequentially planned and executed to insure the best possible outcome for the patient. This case illustrates the successful utilization and primary role of orthodontic treatment by using a new technology system of braces that facilitates the continuation of eruption of maligned teeth into their correct position while inducing alveolar bone remodeling and development after root movement from the onset of the orthodontic therapy. ${ }^{4}$ The benefits of this system are clearly translated in this case and specifically address space management of missing teeth by uprighting an adjacent tooth thereby creating space for the surgical placement and subsequent restoration of a dental implant, the successful management of a constricted maxilla which exhibits localized crossbite and localized rotation and malalignment of teeth and the establishment of a long term stable occlusion and esthetics. The hallmarks of the Fastbraces ${ }^{\circledR}$ system include the simplicity of utilizing a single square wire with a novel bracket design that delivers light forces which may simulate natural eruption forces by uprighting the roots of malpostioned teeth immediately at the onset of treatment. This non-extraction approach not only took about 150 days but also facilitated the remodeling and development of alveolar bone. ${ }^{4}$ This system has been successfully utilized to address non-surgically significant anterior open bites, ${ }^{5}$ severe mandibular prognathism ${ }^{6}$ and adult molar crossbites. ${ }^{7}$ This case report also validates the development of alveolar bone by comparing pre-treatment and post-treatment cross-sectional CBCT images for the maxillary right central incisor which show increased crosssectional alveolar bone development/growth of $1.8 \mathrm{~mm}$. The increase in the buccal-lingual alveolar bone dimension of the maxillary central incisor is clearly evident when measuring the difference between the before apical alveolar bone region dimension and the after apical alveolar bone region dimension which is $7.3 \mathrm{~mm}$ and $9.1 \mathrm{~mm}$, respectively (Figure 13). While this is a limited CBCT sample it does provide radiographic evidence of post-treatment increased crosssectional bone dimension. In addition, comparative measurements of pre and post-treatment casts and photos, show that maxillary inter canine width is increased by $4.5 \mathrm{~mm}$, anterior arch width is increased by $3 \mathrm{~mm}$ and inter premolar width is increased by $1.5 \mathrm{~mm}$ (Figure 14). This post-treatment arch form is the natural arch form the patient would have developed had the teeth erupted properly.

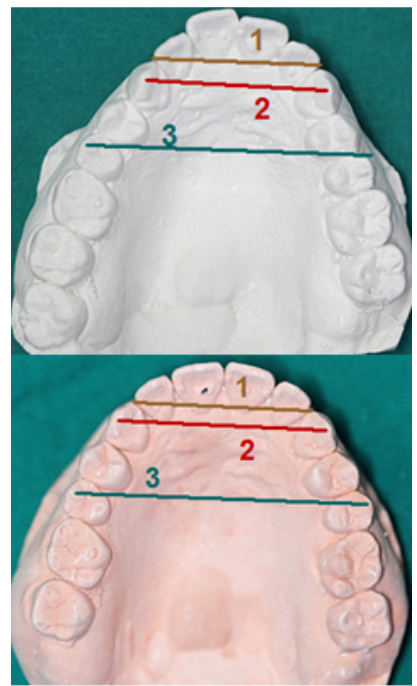

Figure 14 Comparing pre-treatment (white cast) and post-treatment (pink cast) maxillary arch changes. 


\section{Conclusion}

This case report demonstrates the successful outcome of an interdisciplinary adult case with the adjunctive and primary utilization of Fastbraces ${ }^{\circledR}$, a new technology system of braces that facilitates the continuation of eruption of the maligned teeth to correct position while inducing alveolar bone remodeling and development after root movement from the onset of the orthodontic therapy. This case report is the first to provide radiographic validation of favorable alveolar bone remodeling and development. In addition, it illustrates the utilization of a simple, safe and predictable system for the general dentist and specialist to address dental malalignment with esthetic concerns along with dentoalveolar correction for successful implantbased restorative treatment.

\section{Acknowledgments}

None.

\section{Funding}

None.

\section{Conflicts of interest}

The authors declare there is no conflict of interests.

\section{References}

1. Kharbanda OP. Diagnosis and management of malocclusion and dentofacial deformities. 2nd ed. Haryana, India: Elsevier; 2013:1-550.

2. Kokich VG, Spear FM. Guidelines for manging the orthodonticrestorative patient. Semin Orthod. 1997;3(1):3-20.

3. Machuca G, Martinez F, Machuca C, et al. A combination of orthodontic, periodontal and prosthodontic treatment in a case of advanced malocclusion. Intl J Period Rest Dent. 2003;23:499-505.

4. Viazis AD, Viazis E, Pagonis TC. The concept of a new dental disease: orthodontosis and orthodontitis. J Dent Health Oral Disord Ther. 2014;1(5):00030.

5. Viazis AD, Viazis E, Pagonis TC. The fallacy of tongue thrust and nonsurgical treatment of a severe anterior open bite. J Dent Health Oral Disord Ther. 2016;4(4):00120.

6. Viazis AD, Viazis E, Pagonis TC. Non-Surgical Orthodontic Treatment of an Orthognathic Surgical Case. J Dent Health Oral Disord Ther. 2016;4(5):00128.

7. Viazis AD, Viazis E, Pagonis TC. Non-surgical Orthodontic Adult Molar Crossbite Correction and Sleep Apnea. J Dent Health Oral Disord Ther. 2016;5(5):00168. 